\title{
Publisher's Note: Integral for longitudinal phase space tomography on equilibrium distributions \\ [Phys. Rev. ST Accel. Beams 6, 024001 (2003)]
}

Leo Michelotti

(Received 10 March 2003; published 11 March 2003)

DOI: 10.1103/PhysRevSTAB.6.039901

PACS numbers: 41.85.Ew, 45.20.Jj, 99.10.Fg

This manuscript originally appeared in Physical Review Special Topics-Accelerators and Beams online with a corrupted Abstract. This error has been corrected as of 11 March 2003. 